\title{
Comparison of reliability of five patellar position indices at various stifle joint angles in pelvic limbs obtained from cadavers of red foxes (Vulpes vulpes)
}

\author{
James E. Miles, BVetMed; Dorte H. Nielsen, DVM, PhD; Bente R. Jensen, PhD; \\ Jolle Kirpensteijn, DVM, PhD; Eiliv L. Svalastoga, DVM, Dr Med Vet; Thomas Eriksen, DVM, PhD
}

Objective-To compare 5 patellar position indices at various stifle joint angles in cadavers of red foxes, determine measurement reliability, and assess the suitability of these indices for clinical use.

Sample-Pelvic limbs from cadavers of 12 red foxes (Vulpes vulpes).

Procedures-Patellar position in each limb at 7 stifle joint angles ( $30^{\circ}$ to $148^{\circ}$ ) was assessed by use of the Insall-Salvati (IS), modified Insall-Salvati (mIS), de Carvalho (dC), patellotrochlear (PT), and Blackburne-Peel (BP) indices.

Results - Values for all indices varied significantly on the basis of joint angle, but for IS and $\mathrm{mIS}$ indices, this was minor and nonsignificant between $52^{\circ}$ and $130^{\circ}$ and between $52^{\circ}$ and $148^{\circ}$, respectively. The $\mathrm{dC}$ index increased linearly, and PT and BP indices varied polynomially with increases in stifle joint angle. Stifle joint angles measured from radiographs agreed well with the goniometrically set stifle joint angles up to approximately $100^{\circ}$ and diverged thereafter. Intraobserver and interobserver agreement was substantial for all indices, and IS index was the most precise.

Conclusions and Clinical Relevance-IS and mIS index values were effectively independent of stifle joint angle, in contrast to $\mathrm{dC}, \mathrm{PT}$, and BP indices. The BP index varied nonsignificantly across a range of joint angles. To maximize angular accuracy, radiographs should not be obtained at joint angles $>100^{\circ}$. Although dC, PT, and BP indices appeared to be suitable for preoperative and postoperative evaluation of patellar position, BP index appeared to have the most promise for determination of patellar position in clinical applications. (Am J Vet Res 2012;73:263-271)

$\mathbf{M}$ edial patellar luxation is a common orthopedic disorder in dogs. ${ }^{1}$ The relative risk of patellar luxation is greater in small-breed dogs than in large-breed dogs. ${ }^{2}$ Patella alta (patella in a more proximal position than normal) has been identified as a risk factor for recurrent patellar luxation in humans. ${ }^{3-5}$ The patellae of medium- to giant-breed dogs that have medial patellar luxation are more proximally positioned than are patellae of clinically normal dogs. ${ }^{6,7}$ Patella infera (baja), in

Received September 3, 2010.

Accepted December 20, 2010

From the Department of Small Animal Clinical Sciences, Faculty of Life Sciences, Copenhagen University, 1870 Frederiksberg C, Denmark (Miles, Nielsen, Svalastoga, Eriksen); the Department of Exercise and Sport Sciences, Faculty of Science, Copenhagen University, 2200 København N, Denmark (Jensen); and the Department of Clinical Sciences of Companion Animals, Faculty of Veterinary Medicine, Utrecht University, 3584 CS Utrecht, The Netherlands (Kirpensteijn)

The authors thank Malin Jacobsson for technical assistance with preparation of the cadavers and Nils Toft for assistance with the statistical analysis.

Address correspondence to Dr. Miles (jami@life.ku.dk).

\begin{tabular}{|c|c|}
\hline \multicolumn{2}{|r|}{ Abbreviations } \\
\hline BP & Blackburne-Peel \\
\hline $\mathrm{CV}$ & Coefficient of variation \\
\hline $\mathrm{dC}$ & de Carvalho \\
\hline DICOM & $\begin{array}{l}\text { Digital imaging and communications } \\
\text { in medicine }\end{array}$ \\
\hline ICC & Intraclass correlation coefficient \\
\hline $\operatorname{ICC}_{(2, \mathrm{k})}$ & $\begin{array}{l}\text { Case } 2 \text { absolute agreement of intraclass } \\
\text { correlation coefficient }\end{array}$ \\
\hline IS & Insall-Salvati \\
\hline mIS & Modified Insall-Salvati \\
\hline PT & Patellotrochlear \\
\hline
\end{tabular}

which the patella is more distally positioned than normal, is associated with lateral patellar luxation in dogs. ${ }^{7}$ The term patella infera, rather than patella baja, is preferred by some authors. ${ }^{8}$

Studies $^{6,7,9}$ on measurement of the proximodistal patellar position in dogs have focused on the use of the IS index, with or without slight modifications. In contrast, numerous indices have been described for mea- 
surement of the proximodistal position of the patella in humans. ${ }^{10}$ The IS index, as originally described, is the ratio of the lengths of the caudal border of the patellar ligament to the longest diagonal measurement of the patella. ${ }^{11}$ The insertion of the caudal border of the patellar ligament on the tibia is identified as a small notch in both humans ${ }^{11}$ and dogs. ${ }^{9}$ Drawbacks to this technique have been described in the literature of human and veterinary subjects and include variations in nonarticular patellar length, ${ }^{12}$ enthesophytosis of the distal patellar pole,${ }^{6,7}$ masking of the caudal aspect of the patellar ligament by effusion, ${ }^{7}$ masking of the tibial notch by minor axial rotation of the tibia, ${ }^{13}$ and insertion of the caudal border of the patellar ligament proximal to the tibial notch. ${ }^{13}$ Additionally, because this index relies solely on measurements of the patella and patellar ligament, surgical correction for patella alta does not change the index. Consequently, it is not possible to assess the adequacy of surgical correction or identify overcorrection (iatrogenic patella infera) by use of this index.

A modification of the IS index that uses measurements of the distance from the distal articular surface of the patella to the tibial notch and the articular length of the patella has been described in humans. ${ }^{12}$ This modification reduces errors in patient classification attributable to variations in patellar shape and enthesophytosis at the distal pole of the patella that develop when the original IS index calculation is used.

To compare preoperative and postoperative proximodistal patellar position, it is necessary to use indices that describe patellar position relative to the femur or proximal aspect of the tibia. When 2 such indices (BP and Caton-Deschamps indices) are compared with the original and modified IS indices in humans, classification of patellar position (normal vs abnormal) strongly depends on the index that is used. ${ }^{14,15}$ The BP index, which describes the patellar position along a line perpendicular to the tangent of the tibial plateau, ${ }^{16}$ discriminates best among normal, patella alta, and patella infera classifications according to both studies. ${ }^{14,15}$ The Caton-Deschamps index in humans is defined as the ratio of the length of the articular surface of the patella to the distance from the distal end of the articular surface of the patella to the anterosuperior corner of the tibia. ${ }^{17}$ A related index, the $\mathrm{dC}$ index, describes the position of the patella relative to the cranial aspect of the tibial plateau instead of the anteriosuperior corner of the tibia. ${ }^{13}$ Advantages of these 3 indices in humans are that index values do not change with variations in the shape of the patella or morphology near the tibial insertion of the patellar ligament but do potentially change because of surgical treatment.

A method of referencing patellar position relative to the trochlea of the femur by use of magnetic resonance imaging in humans has been reported..$^{18}$ In the PT index, the articular length of the patella is compared with the length of overlap between the articular surface of the patella and the proximal trochlea of the femur when the knee joint is fully extended. In addition to the advantages for the BP, Caton-Deschamps, and $\mathrm{dC}$ indices, the PT index has the advantage that it indicates the relationship between the patella and the trochlea of the femur.
Significant interlimb differences in patellar position index values have been reported in humans. ${ }^{15}$ No differences in mean interlimb index values were identified in a study ${ }^{9}$ of clinically normal large-breed dogs.

Comparisons of various patellar position indices would ideally be performed in a population of animals that are uniform in size and comparable in size to small-breed dogs that are typically affected with medial patellar luxation. Additionally, limb morphology of the animals should be similar to that of domestic dogs; red foxes (Vulpes vulpes) meet these criteria. ${ }^{19}$ The objectives of the study reported here were to compare 5 patellar position indices (IS, mIS, dC, PT, and BP indices) at various stifle joint angles in pelvic limbs of red foxes, determine reliability of measurements used in calculation of these indices, and assess the suitability of these indices for clinical use.

\section{Materials and Methods}

Pelvic limbs were obtained from the cadavers of 12 skeletally mature red foxes ( 6 sexually intact males and 6 sexually intact females). Foxes had been raised on a commercial farm. The foxes were in good physical condition, and no evidence of musculoskeletal disease was observed. Specific approval for this protocol was not required as it was performed by use of cadaver material.

Foxes were euthanized by electrocution (in accordance with Danish $\mathrm{Law}^{20}$ ), ensuring immediate loss of consciousness. After the foxes were euthanized, cadavers were chilled and transported to the Department of Small Animal Clinical Sciences. The cadavers were frozen for storage. Before use, cadavers were thawed to room temperature and limbs were removed by disarticulation of coxofemoral joints and dissection of bones free of musculature. In each limb, the stifle joint capsule, collateral ligaments, patellar ligament, and patella were left in place. The prepared limbs were wrapped in saline $(0.9 \% \mathrm{NaCl})$ solution-soaked gauze swabs and chilled until radiography was performed within 1 to 2 hours after limbs were prepared.

In each limb, a 2.0-mm Kirschner wire ${ }^{a}$ was driven in a lateral to medial direction through the femoral condyles between the origins of the collateral ligaments and cut so that $1 \mathrm{~cm}$ of wire protruded on the lateral aspect. A 1.0-mm Kirschner wire ${ }^{a}$ was inserted in the lateral aspect of the greater trochanter of the femur immediately lateral to the center of the femoral head. A tunnel was drilled in a lateral to medial direction through the calcaneus, and a suture was passed through the tunnel and tied around the femoral neck. A second tunnel was drilled from the trochanteric fossa in a caudal to cranial direction through the femur, and sutures were placed through this tunnel and through the peripatellar fascia, which were used to attach an elastic band along the cranial surface of the femur to ensure the patellar ligament remained taut.

A plastic goniometer a that was accurate to within $2^{\circ}$ was modified by cutting a 1 -mm-wide channel centrally along the long axis of $1 \mathrm{arm}$; this allowed the goniometer to be fitted with the center of rotation over the $2.0-\mathrm{mm}$ wire and the cut arm over the $1.0-\mathrm{mm}$ wire. The stifle joint angle was measured by centering the free arm over the lateral malleolus of the fibula. Adjustment 
of the suture that passed between the femoral neck and calcaneus permitted fixation of the stifle joint at predetermined angles (goniometrically set stifle joint angle).

Digital lateromedial radiographs of each limb were obtained with the stifle joint angle set at $30^{\circ}, 52^{\circ}, 75^{\circ}, 96^{\circ}$, $113^{\circ}, 130^{\circ}$, and $148^{\circ}$. Radiographs included the full length of the femur and tibia and had markers that identified the individual fox, limb, and goniometrically set stifle joint angle. Images were archived as DICOM files.

Anonymity of the DICOM files was accomplished by removing all identification markers and reversing the images of the right limbs to obtain mirror images by use of commercial software. ${ }^{\mathrm{b}}$ The DICOM files were randomly ordered by use of freely available software ${ }^{c}$ such that observers were unaware of the source of each image. Two observers (an experienced veterinary radiologist [DHN] and an experienced veterinary surgeon [JEM]) evaluated each image 3 times by use of freely available software. ${ }^{\mathrm{d}}$ Seven linear measurements and 1 angular measurement were made on each image for determination of the chosen indices (Figure 1).

Except for the BP index, all indices were modified from their original descriptions. The IS index was modified as described elsewhere, ${ }^{7}$ such that the tibial tuberosity was used as the distal endpoint for patellar ligament length, instead of the notch where the caudal border of the ligament inserted into the tibia. The mIS index was modified similarly for consistency. The $\mathrm{dC}$ index was modified by adopting the closest tibial cortex as the distal measurement endpoint instead of restricting it to the cranial end of the tibial plateau. The PT index was modified to include the portion of the trochlea underlying and proximal to the patella, rather than including only the portion of the trochlea underlying the patella. Whereas the knee joint in humans has only a partially engaged patella at the required angle of measurement, the patella of dogs is substantially or completely engaged with the trochlea at the selected angles of measurement. ${ }^{9}$ In addition to indices, stifle joint angle was determined from the radiographs (radiographic stifle joint angle). The center of a circle centered over the femoral condyles served as the vertex of the angle, and the arms of the angle passed through the centers of circles centered over the femoral head and tibiotarsal joint.

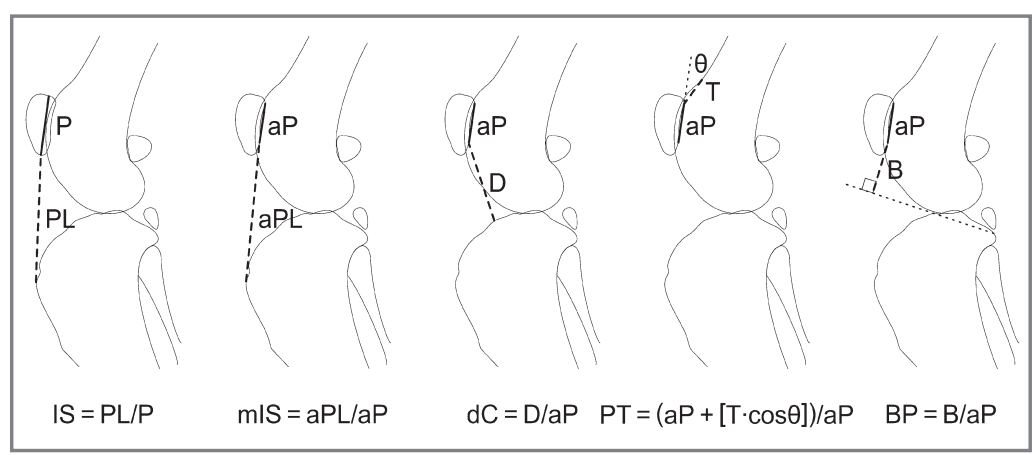

Figure 1-Illustration depicting calculation of the patellar position indices (IS, mIS, $\mathrm{dC}, \mathrm{PT}$, and BP) in stifle joints obtained from cadavers of 12 red foxes (Vulpes vulpes). $\mathrm{aP}=$ Patellar articular surface length. $\mathrm{aPL}=$ Distance from the distal patellar articular surface to the tibial tuberosity. $B=$ Shortest distance from the dista patellar articular surface to a line passing through the cranial and caudal edges of the tibial plateau. D = Distance from the distal patellar articular surface to the closest tibial cortex. $\mathrm{P}=$ Patellar length. $\mathrm{PL}=$ Patellar ligament length from the distal patellar pole to the tibial tuberosity. $T=$ Distance from the proximal articular surface of the patella to the proximal trochlea. $\theta=$ Angle between the line of the patellar articular surface and a line extending from the proximal articular surface of the patella to the proximal femoral trochlea.
Statistical analysis-For each observation, data for calculation of the 5 indices and values of measured radiographic stifle joint angle were collected, along with the goniometrically set stifle joint angle, limb, observer, observation number, sex of fox, and fox. Indices for each observation by each observer, mean indices for each observer, and mean indices for both observers combined were calculated. Data were reported as mean \pm SD unless otherwise stated. Values were considered significant at $P<0.05$.

Statistical analysis was performed by use of statistical software. ${ }^{e}$ Data were determined to be normally distributed by use of quantile-quantile plots, residual plots, and the Shapiro-Wilk test, and variances were compared visually by use of box plots. The $\operatorname{ICC}_{(2, \mathrm{k})}$ and associated $95 \%$ confidence limits for the intraobserver

Table 1-Calculated reference intervals and regression variables for each goniometrically set joint angle $(\theta)$ for 5 patellar position indices determined in stifle joints obtained from cadavers of 12 red foxes (Vulpes vulpes).

\begin{tabular}{|c|c|c|c|c|c|}
\hline Variable & IS & $\mathrm{mlS}$ & dC & PT & BP \\
\hline \multicolumn{6}{|l|}{ Joint angle $\left({ }^{\circ}\right)^{*}$} \\
\hline 30 & $1.8-2.4$ & $2.9-3.9$ & $0.7-1.3$ & $1.8-2.5$ & $1.1-1.7$ \\
\hline 52 & $1.9-2.5 \dagger$ & $2.9-4.1 \dagger$ & $0.9-1.7$ & $2.1-2.8 \dagger$ & $1.2-2.0$ \\
\hline 75 & $1.9-2.5 \dagger$ & $2.9-4.1 \dagger$ & $1.2-2.0$ & $2.1-2.8 \dagger$ & $1.4-2.2 \dagger$ \\
\hline 96 & $1.9-2.5 \dagger$ & $2.9-4.1 \dagger$ & $1.4-2.3$ & $2.1-2.8 \dagger$ & $1.4-2.2 \dagger$ \\
\hline 113 & $1.9-2.5 \dagger$ & $2.9-4.1 \dagger$ & $1.6-2.4$ & $1.7-2.5$ & $1.4-2.2 \dagger$ \\
\hline 130 & $1.9-2.5 \dagger$ & $2.9-4.1 \dagger$ & $1.8-2.6$ & $1.4-2.2$ & $1.4-2.1$ \\
\hline 148 & $1.9-2.6$ & $2.9-4.1 \dagger$ & $1.9-2.8$ & $1.0-1.8$ & $1.3-2.1$ \\
\hline Regression & $(0.00095 \bullet \theta)+2.1$ & $(0.0013 \bullet \theta)+3.4$ & $(0.011 \bullet \theta)+0.71$ & $(0.024 \bullet \theta)-\left(0.00017 \bullet \theta^{2}\right)+1.6$ & $(0.011 \bullet \theta)-\left(0.00005 \bullet \theta^{2}\right)+1.2$ \\
\hline$P$ value & 0.003 & 0.046 & $<0.001$ & $<0.001$ & $<0.001$ \\
\hline$R^{2}$ & 0.051 & 0.024 & 0.840 & 0.790 & 0.190 \\
\hline \multicolumn{6}{|l|}{$95 \%$ confidence interval } \\
\hline Linear regression & 0.00032 to 0.00157 & 0.00002 to 0.00256 & 0.01027 to 0.01172 & 0.01988 to 0.02732 & 0.00949 to 0.01280 \\
\hline Polynomial regression & - & 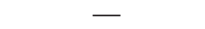 & - & -0.00015 to -0.00019 & -0.00004 to -0.00006 \\
\hline
\end{tabular}

*Values reported are $95 \%$ reference intervals. WWithin an index, values did not differ significantly $(P \geq 0.05)$ between adjacent joint angles. - = Not calculated. 

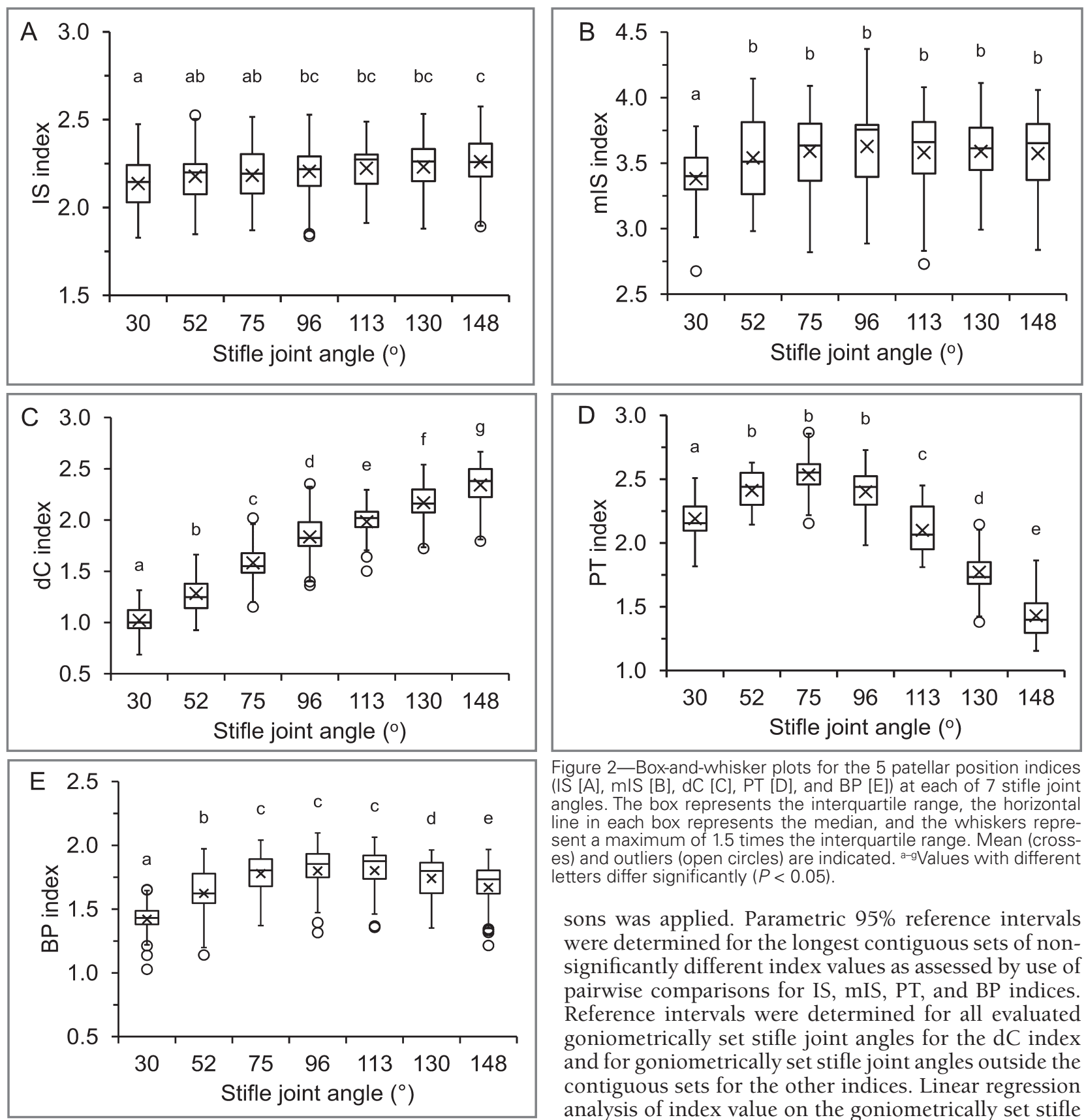

$(\mathrm{k}=3)$ and interobserver $(\mathrm{k}=2)$ comparisons were calculated $^{21}$ and interpreted ${ }^{22}$ as previously described. Because interobserver and intraobserver comparisons for the IS index in dogs have good agreement, ${ }^{7,9}$ the other selected indices were compared for feasibility and intraobserver and interobserver reliability by use of the IS index as a standard. Coefficients of variation were calculated for comparison of index reliability ${ }^{23}$ because 2 indices ( $\mathrm{mIS}$ and $\mathrm{dC}$ ) had evidence of heteroscedasticity on evaluation of plots of SD against the mean and by use of a White test.

The effect of the goniometrically set stifle joint angle on index value was investigated by use of a mixedmodel ANOVA that had goniometrically set stifle joint angle, limb, and sex as fixed effects and fox as a random effect. A Bonferroni correction for multiple compari-

Figure 2-Box-and-whisker plots for the 5 patellar position indices (IS [A], mIS [B], dC [C], PT [D], and BP [E]) at each of 7 stifle joint angles. The box represents the interquartile range, the horizontal line in each box represents the median, and the whiskers represent a maximum of 1.5 times the interquartile range. Mean (crosses) and outliers (open circles) are indicated. ${ }^{a-g}$ Values with different letters differ significantly $(P<0.05)$.

sons was applied. Parametric 95\% reference intervals were determined for the longest contiguous sets of nonsignificantly different index values as assessed by use of pairwise comparisons for IS, mIS, PT, and BP indices. Reference intervals were determined for all evaluated goniometrically set stifle joint angles for the $\mathrm{dC}$ index and for goniometrically set stifle joint angles outside the contiguous sets for the other indices. Linear regression analysis of index value on the goniometrically set stifle joint angle was performed for IS, mIS, and dC indices, and polynomial regression analysis was performed for $\mathrm{PT}$ and $\mathrm{BP}$ indices.

Radiographic stifle joint angle was assessed by use of a mixed-model ANOVA that had limb (left vs right) and sex of fox as fixed effects and fox as a random effect; results were grouped by the goniometrically set stifle joint angle. Radiographic stifle joint angles and goniometrically set stifle joint angles were further compared by use of linear regression analysis and a BlandAltman plot. ${ }^{24}$

\section{Results}

The values of all patellar position indices changed significantly $(P<0.001)$ with joint angle, although the smallest changes were seen in IS and mIS indices 
Table 2-Mean \pm SD interlimb differences (ILD) for 5 patellar position indices determined in stifle joints obtained from cadavers of 12 red foxes.

\begin{tabular}{lcccc}
\hline Variable & IS & mIS & dC & BP \\
\hline Mean ILD & $0.03 \pm 0.10$ & $0.03 \pm 0.23$ & $0.04 \pm 0.12$ & $0.004 \pm 0.21$ \\
ILD (\%) & 1.2 & 0.9 & 0.3 & $0.01 \pm 0.09$ \\
Mean absolute ILD & $0.08 \pm 0.06$ & $0.18 \pm 0.14$ & $0.10 \pm 0.08$ & 0.1 \\
Absolute ILD (\%) & 3.8 & 5.0 & 5.8 & $0.16 \pm 0.13$ \\
Predicted mean ILD & $0.002 \pm 0.003$ & $0.002 \pm 0.004$ & $0.019 \pm 0.035$ & $0.002 \pm 0.003$ \\
Predicted mean absolute ILD & $0.003 \pm 0.002$ & $0.004 \pm 0.003$ & $0.032 \pm 0.024$ & $0.003 \pm 0.002$ \\
\hline
\end{tabular}

The ILD was calculated as the mean of the index value for the right limb minus the index value for the left limb for each of the 12 foxes. Absolute ILD was calculated without regard to the sign (positive or negative) of the difference. Percentage values are relative to the overall index mean. Predicted mean ILD and absolute ILD values were generated by use of the regression equations for each index and radiographic stifle joint angle values for each limb.

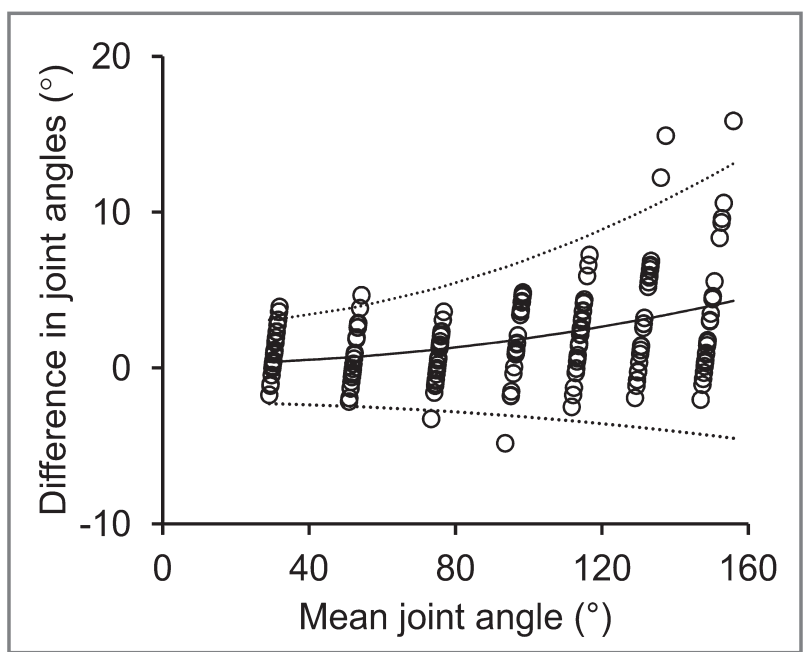

Figure 3-Bland-Altman plot comparing the radiographic stifle joint angle with the goniometrically set stifle joint angle. Differences between the 2 angles (radiographic stifle joint angle - goniometrically set stifle angle) are plotted against the mean of the 2 angles. Each circle represents the difference between the 2 angles for 1 stifle joint. The mean difference or bias (solid line) and the $95 \%$ limits of agreement (dotted lines) are indicated.

(Figure 2). For the $\mathrm{dC}$ index, all adjacent pairs of angles had significantly different mean index values, whereas for the other indices, sets of at least 3 adjacent angles could be identified for which the mean index values were not significantly different from each other. Calculated reference intervals and regression variables were summarized (Table 1).

A significant effect of limb was observed for IS $(P=$ $0.006)$ and $\mathrm{dC}(P=0.009)$ index values but not for mIS $(P=0.21)$, PT $(P=0.86)$, or BP $(P=0.29)$ index values. Index values obtained for the right limb were slightly greater than those for the left limb for all indices at all goniometrically set stifle joint angles, except for the mIS index at $52^{\circ}$; PT index at $75^{\circ}, 113^{\circ}, 130^{\circ}$, and $148^{\circ}$; and $\mathrm{BP}$ index at $113^{\circ}$ and $130^{\circ}$. Ratios for the largest index values between the limbs (left stifle joint-to-right stifle joint) for IS, mIS, dC, PT, and BP indices were 32:52, $38: 46,31: 53,35: 49$, and 33:51, respectively. In all instances, the mean interlimb differences in index values were small, although absolute differences (regardless of whether the sign of the difference was positive or negative) between the limbs were larger. Mean differences, mean absolute differences, and percentage differences of the index values were summarized (Table 2).

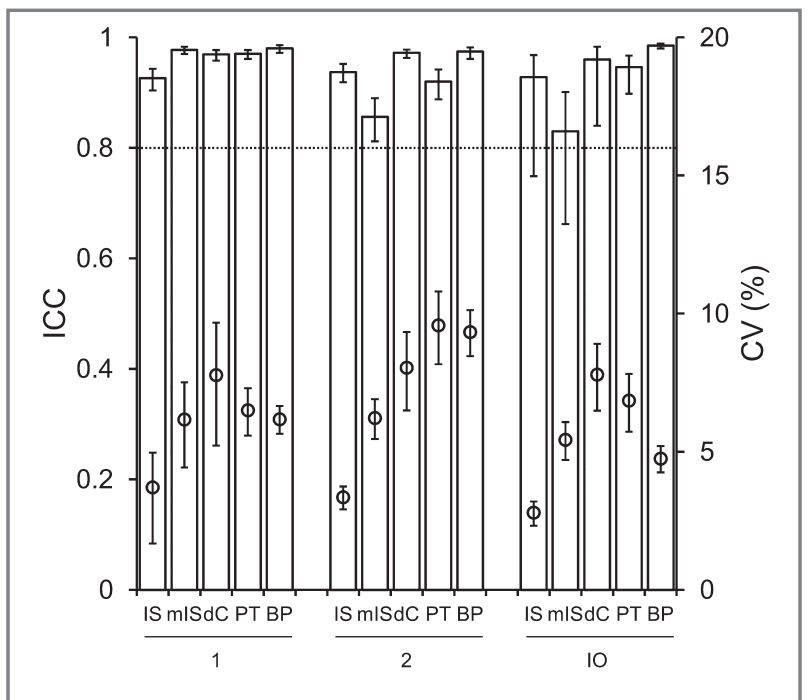

Figure 4-Interobserver (IO) and intraobserver reliability of measurements for 2 observers (1 and 2) and 5 indices (IS, $\mathrm{mIS}, \mathrm{dC}$, PT, and BP) of the stifle joint. Results reported are the ICCs (bars) and the CVs (circles). Error bars represent the $95 \%$ confidence intervals. The cutoff point for substantial ICC agreement as defined elsewhere ${ }^{21}$ is indicated (dotted line).

Linear regression analysis revealed that the radiographic stifle joint angle was significantly $(P<0.001)$ related to the goniometrically set stifle joint angle, with a mean \pm SE gradient of $1.026 \pm 0.0057\left(R^{2}, 0.995\right)$. Radiographic and goniometrically set stifle joint angles were also compared by use of linear regression analysis and a Bland-Altman plot (Figure 3). The radiographic stifle joint angle had a bias of $0.4^{\circ}$ to $4.3^{\circ}$, compared with the goniometrically set stifle joint angle, with limits of agreement increasing from $-2^{\circ}$ to $3^{\circ}$ (range, $5^{\circ}$ ) to $-5^{\circ}$ to $13^{\circ}$ (range, $18^{\circ}$ ).

The radiographic stifle joint angle was significantly greater in radiographs of the right limb than the left limb for goniometrically set stifle joint angles of $96^{\circ}$ $(P=0.049), 113^{\circ}(P=0.001)$, and $130^{\circ}(P=0.034)$. At all angles evaluated, the radiographic stifle joint angle of the right limb was slightly greater than that of the left limb, but these differences were small (1.7 $\pm 1.0^{\circ}$ ). Index values were predicted for all indices and angles for left and right limbs by use of regression analysis data, and the mean differences and mean absolute differences of index values between limbs were calculated. Differences in index values between left versus right stifle joints were smaller for predicted in- 
dex values than for index values that were calculated from measurement of radiographs (Table 2). When the interlimb differences were corrected for the calculated differences (because of the influence of the radiographic stifle joint angle), the original pattern of ratios for the index values was maintained with corrected index value ratios (left stifle joint-to-right stifle joint) of 38:46, 37:47, 37:47, 35:49, and 35:49 for IS, mIS, dC, PT, and BP indices, respectively.

Sex of fox did not significantly affect IS $(P=0.51)$, mIS $(P=0.42), \mathrm{dC}(P=0.88), \mathrm{PT}(P=0.81)$, and BP $(P$ $=0.68)$ indices. Intraobserver and interobserver agreements were substantial as assessed by use of ICC ${ }_{(2, \mathrm{k})}$ values, which indicated that the measurement techniques were both repeatable and reproducible (Figure 4). Significant differences were detected between various indices for both observers, but these differences were considered minor because all values were greater than the previously defined threshold of $0.8 .^{21}$ The $\operatorname{ICC}_{(2, \mathrm{k})}$ values for the interobserver comparison were similar to but had greater variability than the intraobserver values. Values for both the mIS and PT indices were significantly lower than those for the BP index, but no other significant differences between interobserver ICC values were detected. The interobserver CVs for IS index were significantly $(P<0.001)$ lower than the interobserver ICs for the other 4 indices, which indicated better precision for the IS index than for indices that referenced the articular surface (mIS, dC, PT, and BP indices). Values for mIS and BP indices did not differ significantly, but values for both indices were significantly lower than values for the $\mathrm{dC}$ and PT indices.

\section{Discussion}

Key findings in the study reported here were the relative insensitivity to stifle joint angle of IS and mIS indices and the linear ( $\mathrm{dC}$ index) and polynomial (PT and BP indices) relationships of the other indices to stifle joint angle. All indices had substantial agreement within and between observers.

The indices evaluated were selected because they had been previously used in dogs (IS index) or because we believed they were of potential value and could be readily applied in clinical veterinary practice. Four of the indices were modified slightly from their original descriptions to simplify their use and application in clinical veterinary practice.

Goniometrically set stifle joint angles were chosen on the basis of stifle joint angles found during walking and trotting in clinically normal dogs. ${ }^{9}$ We extended the range of angles for flexion of the stifle joint to include angles that might be found during the transition from standing to sitting in dogs.

Pelvic limbs of red foxes were used in the present study because red foxes are similar in size to smallbreed dogs that are typically affected with medial patellar luxation, their limb morphology is similar to that of domestic dogs, ${ }^{19}$ and there is little difference in size among individual animals (which reduced interindividual variation in measurements). Furthermore, the readily available cadaver specimens made red foxes ideal for this study.
The IS index value is generally considered to be independent of joint angle in humans, which has the advantage that radiography does not need to be performed at a defined joint angle. ${ }^{12}$ Results of studies indicating both independence ${ }^{9}$ and curvilinear dependence ${ }^{7}$ of IS index values and stifle joint angle in dogs have been reported. In contrast to results of those studies, ${ }^{7,9}$ results of the present study indicated a weak linear effect of stifle joint angle on the IS index value; these results can be interpreted as supportive of the findings of stifle joint angle independence ${ }^{9}$ (because the IS index value was not affected to a clinically important degree by clinically relevant stifle joint angles since the regression gradient was minimally different from zero). Examination of radiographs of fox stifle joints in the present study indicated that the patellar ligament increasingly wrapped around the cranioproximal aspect of the tibia as stifle flexion was increased. Because patellar ligament length (Figure 1) was measured directly between the distal pole of the patella and the tibial tuberosity, the influence of stifle joint angle was expected in this index. This influence of stifle joint angle appeared more pronounced with the mIS index but (similar to its parent IS index), the regression line gradient was small, and index values were almost unaffected by stifle joint angle. Variability in data distribution for the mIS index was greater and reference ranges wider than for the other indices evaluated, and it therefore seems unlikely that use of the mIS index would have substantial advantages over use of the IS index, at least in dogs that do not have patellar abnormalities.

The dC index had a strong positive linear relationship with stifle joint angle, and there were significant differences between mean $\mathrm{dC}$ index values at all stifle joint angles in the present study. For use in a clinical setting, radiographs would have to be obtained at a set angle for which the reference interval had already been defined or the reference interval would have to be predicted for the angle at which the radiograph was obtained. In each case, a means of determining the stifle joint angle from a lateral radiograph would be required to confirm the accuracy of the set stifle joint angle or to calculate the reference interval. Although a technique for determination of the joint angle from lateral radiographs of the stifle joint has been described in dogs, ${ }^{7}$ it is uncertain how angles measured by use of those criteria relate to the angle-setting technique we used or to the radiographic stifle joint angle we measured in the present study. Similar caveats apply to PT index values, which had a more complex polynomial relationship with stifle joint angle but were least variable between stifle joint angles from $52^{\circ}$ to $96^{\circ}$. Although the relationship of BP index values to stifle joint angles was also polynomial, variation in BP index values because of changes in joint angle was much less than for $\mathrm{dC}$ or PT index values. This finding suggests potentially increased clinical usefulness of the BP index because accuracy of determination of the stifle joint angle would be less important for this index, at least within a distinct range of stifle joint angles $\left(75^{\circ}\right.$ to $\left.113^{\circ}\right)$ used in the present study. Use of angles in this range would have the secondary advantage of increasing the likelihood that the patellar tendon would remain taut in clinical 
patients, which is a prerequisite for accurate calculation of all 5 indices.

Mean \pm SD values of the IS index in clinically normal large-breed dogs ( $1.68 \pm 0.13 ; 95 \%$ reference interval, 1.33 to 2.03$)^{9}$ and a mixed population of clinically normal dogs $(2.02 \pm 0.2 ; 95 \%$ reference interval, 1.62 to $2.42)^{7}$ have been reported. The IS index values obtained in the present study (1.9 to 2.5) compare favorably with those of the previous study ${ }^{7}$ on which our measurement criteria were based; these findings suggest a diagnosis of patella alta would be made at IS index values $>2.5$ and patella infera at IS index values $<1.9$. Differences between the IS values reported in the 2 previous studies $^{7,9}$ can in part be explained by the definition used for the index measurements. We found that use of the tibial notch rather than the tibial tuberosity as the distal landmark for patellar ligament length reduced IS index values by approximately 13\% (data not shown). We do not believe that this fully explains the difference between our results and those of another study. ${ }^{9}$ The remainder of the difference may be attributable to differences in species or body size.

The cause of the observed differences in stifle joint angle between the left and right limbs in the present study was unclear. Radiographic images were reversed to provide mirror images to remove observer bias during measurement. Therefore, possible causes included a true anatomic difference in femoral form or ligament insertion, a limb bias during placement of the marker Kirschner wires before radiography, or a bias in the goniometer used for establishing limb angle during radiography. The mean angular difference was within the limits of accuracy for the goniometer.

Another study ${ }^{9}$ in which researchers investigated use of the IS index in dogs did not identify any differences in mean index values between left and right stifle joints. Significant differences in the mean IS index values between left and right stifle joints that were identified in the present study were clinically unimportant. In contrast, the absolute interlimb differences were much larger. This disparity between the mean differences and absolute differences implies that comparison of mean values for interlimb differences may be simplistic and could yield misleading results. The interlimb differences cannot be explained simply by the differences in radiographic stifle joint angle versus goniometrically set stifle joint angle between the left and right limbs because this had only a minor effect on index value. It is unclear how these differences would affect clinical interpretation, although it seems more likely that each of a patient's limbs could be classified in a different diagnostic category (normal, alta, or infera) with mIS or PT indices than with IS, dC, or BP indices.

All 5 indices had a frequency bias toward right limbs having a greater index value than left limbs, which may indicate an influence of limb dominance in stifle joint anatomy of red foxes. This apparent dominance effect persisted even after correction of the data for the observed differences in stifle joint angle. It has been suggested that most clinically normal dogs could be classified as right-limb dominant. ${ }^{25}$

The radiographic stifle joint angle, as defined in the study reported here, had a mean value that was $1^{\circ}$ great- er than the goniometrically set stifle joint angle at angles up to approximately $100^{\circ}$, but values diverged more at higher angles. This difference reflects the choice of the vertices used in the 2 methods performed to set or determine stifle joint angle. A Kirschner wire driven through the origins of the collateral ligaments was used as the vertex of the goniometrically set stifle joint angle, and the center of a circle centered over the femoral condyles was used as the vertex for calculation of the radiographic stifle joint angle. We chose to measure the radiographic stifle joint angle as defined here rather than measuring between the Kirschner wires because although the wires were accurately located on the lateral aspect of the limb, the location of the wire on the medial aspect of the bone was different in some limbs. This resulted in an oblique projection of Kirschner wires on these radiographs, and compensation during angle measurement was not possible.

Evaluation of the ICC values indicated that intraobserver and interobserver agreements were substantial for all 5 indices examined. Agreement was not affected by experience of the radiographers. Intraobserver ICCs differed among indices, but these differences were clinically unimportant because all values were greater than the previously defined cutoff value of $0.80 .^{22}$ The slightly lower interobserver agreement values suggested that the 2 observers consistently selected slightly different endpoints for most measurements. Although we expected the IS index to have superior ICC values, this was not the case. The ICC relates between-subject variability and within-subject variability in such a way that if between-subject variability decreases, then the within-subject variability (or error component) becomes mathematically more important ${ }^{26}$ Because values of the $\mathrm{dC}, \mathrm{PT}$, and BP indices varied more than did values of the IS and mIS indices with changes in stifle joint angle, the error components of the ICC were less important for the $\mathrm{dC}, \mathrm{PT}$, and BP indices than for the IS and mIS indices.

Evaluation of the $\mathrm{CV}$, an alternate method of assessing measurement precision, clearly revealed that the IS index was significantly more precise than were the indices referencing articular surfaces. This is probably attributable to the low subjectivity involved in measuring the index components of patellar length and patellar ligament length from the distal patellar pole to the tibial tuberosity. Notably, measurement of articular length was more subjective. Although most limbs had a single concave articular facet, several were identified that had a biconcave facet, which made it more difficult to precisely identify the distal extent of a joint surface. In these cases, the observers relied on changes in bone density to identify the presumed extent of the patellar articular surface. Misidentification of the true distal endpoint of the patellar articular surface has implications in the determination of the distance from the distal articular surface to the tibial tuberosity and the distance from the distal articular surface to the closest tibial cortex. Mathematically, this interdependence in determination of values tends to be proportionately more important when the denominator and numerator of the fraction are of similar magnitude. Thus, small disagreements between observers in patellar articular 
surface length would be expected to have a greater influence on the $\mathrm{CV}$ for the $\mathrm{dC}$ index than for the mIS index, as was indicated by our results. It was the observers' impression that determination of the PT index was extremely subjective relative to the other measured indices because of difficulties in precisely identifying the endpoint of the proximal trochlea of the femur; however, this impression was not supported by the ICCs or CVs. This unexpected reliability of the PT index may in part be attributable to the fact that the proximal end of the patellar articular surface was more clearly defined than was the distal end, which avoided an interdependence problem between length of the patellar articular surface and distance from the proximal articular surface of the patella to the proximal trochlea of the femur. Intraobserver CVs for the BP index differed markedly. Studies ${ }^{27,28}$ have consistently identified observer variation in determination of the tibial plateau angle in dogs. At stifle joint angles $>75^{\circ}$, the intersection of the shortest line from the distal articular surface to a line passing through the cranial and caudal edges of the tibial plateau and the line of the tibial plateau was cranial to the tibial plateau itself; therefore, errors in the identification of landmarks could have greater impact at higher stifle joint angles. Assessment of the effect of tibial plateau landmark identification on BP index reliability in dogs needs to be determined.

It is difficult to perform absolute comparisons of our interobserver and intraobserver reliability findings with those in studies ${ }^{14,15}$ on humans because different measures of reliability (Spearman rank correlation coefficient and SE calculation) were used in the human studies. In general, the IS index was better than the mIS index and the Caton-Deschamps index (the index most similar to the dC index used in the present study) was better than the IS index in one study ${ }^{15}$ and worse in the other study. ${ }^{14}$ In both of the aforementioned studies, ${ }^{14,15}$ use of the BP index yielded results that had the best intraobserver and interobserver agreement, compared with results for the other indices.

To our knowledge, there are no preexisting studies on values for the mIS, dC, PT, or BP indices in the veterinary literature. Each of the patellar position indices had strengths and weaknesses. Although the IS and mIS indices were largely independent of the effect of stifle joint angle and therefore required no particular precautions when obtaining or interpreting radiographs, both would be insensitive to surgical movement of the tibial tuberosity. The mIS index was slightly less reliable than the IS index but would be less likely to be affected by degenerative joint changes that can accompany patellar luxation. In contrast, values of the $\mathrm{dC}, \mathrm{PT}$, and $\mathrm{BP}$ indices were dependent on stifle joint angle. Therefore, preoperative and postoperative radiographic comparisons must be performed with care to avoid shifting the reference interval because of changes in the stifle joint angle. However, all 3 indices can be used to compare patellar height after surgical movement of the tibial tuberosity. Whereas a study ${ }^{15}$ in humans indicated that degenerative joint changes enhance reliability for identification of the patellar articular surface, which might aid in the use $\mathrm{dC}$ and BP indices, there is a risk that degenerative changes along the proximal trochlear ridges of the femur may obscure landmarks for correct assessment of the PT index. In addition, determination of the PT index was not necessarily straightforward because measurement of both linear distance and angulation was required for calculating the length of the trochlea that was in contact with and proximal to the articular surface of the patella. Although the BP index was less affected by stifle joint angle than were the $\mathrm{dC}$ or PT indices and interobserver reliability for the BP index was good in the present study, variations in tibial plateau angles among dog breeds ${ }^{29,30}$ may cause difficulties in establishing reference intervals for the BP index.

Further research is needed to determine reliability of patellar position indices, effect of stifle joint angle on measurements made for calculation of indices, and effects of variations in tibial plateau angle before diagnostic reliability of use of the indices in dogs can be assessed. It seems appropriate to focus future studies of patellar position index on joint angles $<100^{\circ}$ because there is good agreement between the 2 methods of determining stifle joint angle used in the study reported here (goniometrically set and radiographic stifle joint angle) and the patellar tendon is more likely to remain taut at these angles.

Of the 5 indices examined in the present study, values of 2 indices (IS and mIS) were largely independent of joint angle and values of 3 indices (dC, PT, and BP) were not. For clinicians, the choice of an appropriate index for ascertaining proximodistal patellar position represents a dilemma. In this study, we detected substantial intraobserver and interobserver reliability for all measurements. In terms of suitability for postoperative use, the dC, PT, and BP indices appeared to be superior to the IS and mIS indices but had lower intraobserver and interobserver reliability of measurement, compared with those for the IS index. For clinical use, a reliable technique for determination of stifle joint angle is required for $\mathrm{dC}, \mathrm{PT}$, and BP indices, although the latter had the least variation with changes in the joint angle. Consequently, despite slightly lower precision of the BP index, compared with precision of the IS index, the BP index would probably be the simplest method to apply in clinical practice for preoperative and postoperative assessment of patellar position.

\footnotetext{
a. Veterinary Instrumentation, Sheffield, South Yorkshire, England.

b. Sante DICOM Editor, Santesoft, Athens, Greece.

c. Ant Renamer, Antoine Potten, Brussels, Belgium. Available at: www.antp.be/software/renamer. Accessed Oct 13, 2011.

d. Sante DICOM Viewer, version 1.4, Santesoft, Athens, Greece.

e. SAS, version 9.1, SAS Institute Inc, Cary, NC.
}

\section{References}

1. Piermattei DL, Flo GF, Decamp CE. The stifle joint. In: Handbook of small animal orthopedics and fracture repair. 4th ed. St Louis: Saunders Elsevier, 2006;562-632.

2. Priester WA. Sex, size, and breed as risk factors in canine patellar dislocation. J Am Vet Med Assoc 1972;160:740-742.

3. Insall J, Salvati E, Goldberg V. Recurrent dislocation and highriding patella. Clin Orthop 1972;88:67-69.

4. Simmons E, Cameron JC. Patella alta and recurrent dislocation of the patella. Clin Orthop 1992;274:265-269.

5. Aglietti P, Insall JN, Cerulli G. Patellar pain and incongruence. 1: measurements of incongruence. Clin Orthop 1983;176:217-224. 
6. Johnson AL, Broaddus KD, Hauptman JG, et al. Vertical patellar position in large-breed dogs with clinically normal stifles and large-breed dogs with medial patellar luxation. Vet Surg 2006;35:78-81.

7. Mostafa AA, Griffon DJ, Thomas MW, et al. Proximodistal alignment of the canine patella: radiographic evaluation and association with medial and lateral patellar luxation. Vet Surg 2008;37:201-211

8. Grelsamer RP. Patellar nomenclature: the tower of babel revisited. Clin Orthop 2005;436:60-65.

9. Johnson AL, Probst CW, Decamp CE, et al. Vertical position of the patella in the stifle joint of clinically normal large-breed dogs. Am J Vet Res 2002;63:42-46.

10. Teitge RA. Plain patellofemoral radiographs. Oper Tech Sport Med 2001;9:134-151.

11. Insall J, Salvati E. Patella position in the normal knee joint. $R a$ diology 1971;101:101-104.

12. Grelsamer RP, Meadows S. The modified Insall-Salvati ratio for assessment of patellar height. Clin Orthop 1992;282:170176 .

13. de Carvalho A, Andersen AH, Topp S, et al. A method for assessing the height of the patella. Int Orthop 1985;9:195-197.

14. Seil R, Muller B, Georg T, et al. Reliability and interobserver variability in radiological patellar height ratios. Knee Surg Sports Traumatol Arthrosc 2000;8:231-236.

15. Berg EE, Mason SL, Lucas MJ. Patellar height ratios-comparison of four measurement methods. Am J Sports Med 1996;24:218-221.

16. Blackburne JS, Peel TE. New method of measuring patellar height. J Bone Joint Surg Br 1977;59:241-242.

17. Caton J, Deschamps G, Chambat P, et al. Patella infera. Apropos of 128 cases [in French]. Rev Chir Orthop Reparatrice Appar Mot 1982;68:317-325.

18. Biedert RM, Albrecht S. The patellotrochlear index: a new in- dex for assessing patellar height. Knee Surg Sports Traumatol Arthrosc 2006;14:707-712.

19. Wayne RK. Limb morphology of domestic and wild canids-the influence of development on morphological change. J Morphol 1986;187:301-319.

20. Danish Law. nr 583 Order on the slaughter and euthanasia of animals, chapter $11, \S 56$ and $\$ 59$. Available at: www.retsinformation. dk/Forms/R0710.aspx?id=2649\#K11. Accessed Oct 13, 2011.

21. Shrout PE, Fleiss JL. Intraclass correlations-uses in assessing rater reliability. Psychol Bull 1979;86:420-428.

22. Shrout PE. Measurement reliability and agreement in psychiatry. Stat Methods Med Res 1998;7:301-317.

23. Bland JM, Altman DG. Statistics notes: measurement error proportional to the mean. BMJ 1996;313:106.

24. Bland JM, Altman DG. Measuring agreement in method comparison studies. Stat Methods Med Res 1999;8:135-160.

25. Colborne GR. Are sound dogs mechanically symmetric at trot? No, actually. Vet Comp Orthop Traumatol 2008;21:294-301.

26. Streiner DL, Norman GR. "Precision" and "accuracy": two terms that are neither. J Clin Epidemiol 2006;59:327-330.

27. Caylor KB, Zumpano CA, Evans LM, et al. Intra- and interobserver measurement variability of tibial plateau slope from lateral radiographs in dogs. J Am Anim Hosp Assoc 2001;37:263-268.

28. Fettig AA, Rand WM, Sato AF, et al. Observer variability of tibial plateau slope measurement in 40 dogs with cranial cruciate ligament-deficient stifle joints. Vet Surg 2003;32:471-478.

29. Morris E, Lipowitz AJ. Comparison of tibial plateau angles in dogs with and without cranial cruciate ligament injuries. J Am Vet Med Assoc 2001;218:363-366.

30. Wilke VL, Conzemius MG, Besancon ME, et al. Comparison of tibial plateau angle between clinically normal Greyhounds and Labrador Retrievers with and without rupture of the cranial cruciate ligament. J Am Vet Med Assoc 2002;221:1426-1429. 